\title{
On Asymptotic Posterior Normality for Stochastic Processes
}

\author{
BY \\ C. C. HEYDE and I. M. JOHNSTONE
}

Reprinted from

THE JOURNAL OF THE ROYAL STATISTICAL SOCIETY

SERIES B (METHODOLOGICAL)

Volume 41, No. 2, 1979

(pp. 184-189)

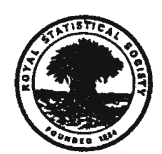

PRINTED FOR PRIVATE CIRCULATION

1979 


\title{
On Asymptotic Posterior Normality for Stochastic Processes
}

\author{
By C. C. HeYde and \\ I. M. JOHNSTONE \\ CSIRO, Canberra \\ Australian National University
}

[Received August 1978. Revised October 1978]

\begin{abstract}
SUMMARY
Asymptotic normality of the posterior distribution of a parameter in a stochastic process is shown to hold under conditions which do little more than ensure consistency of a maximum likelihood estimator. Much more stringent conditions are required to ensure asymptotic normality of the MLE. This contrast, which has implications of considerable significance, does not emerge in the classical context of independent and identically distributed observations.
\end{abstract}

Keywords: PARAMETER ESTIMATION; STOCHASTIC PROCESSES; BAYESIAN METHODS; MAXIMUM LIKELIHOOD ESTIMATOR; ASYMPTOTIC NORMALITY

\section{INTRODUCTION}

IN the classical context of independent and identically distributed observations it is well known that, under mild conditions, the maximum likelihood estimator (MLE) is consistent and, with appropriate normalization, asymptotically normally distributed. This "regular" behaviour is much less common when one moves to the more general context of estimation for stochastic processes. Indeed, quite stringent conditions are required to ensure that the MLE, when appropriately normalized, has an asymptotically normal distribution; e.g. Basawa et al. (1976), Heyde (1978).

Interestingly, a Bayesian approach to the problem has much greater universality. The conditions for asymptotic normality of the posterior distribution, appropriately scaled, are very similar to those which ensure consistency of the MLE and are much weaker than those required for asymptotic normality of the MLE. This contrast does not appear in the i.i.d. case, where the conditions for asymptotic normality of the MLE simplify to (essentially) a finite variance requirement and hence it appears to have escaped the attention which it deserves. Our methodology is similar to that of Walker (1969) who has discussed the i.i.d. case but the conditions have been simplified.

A Bayesian approach to some questions of "hypothesis testing" in a large sample situation also has an important advantage. It can provide, via the posterior distribution, a single categorical answer in each application while Neyman-Pearson based methodology can, in many cases, lead to complex questions of optimality of procedure in which categorical answers do not seem possible; e.g. Basawa and Scott (1976, 1977), Feigin (1978), Sweeting (1978).

\section{The Setting AND Results}

Let $\left(X_{1}, X_{2}, \ldots, X_{n}\right)$ be a sample of consecutive observations from a stochastic process whose distribution depends on a single unknown parameter $\theta \in \Theta$, $\Theta$ denoting an interval. We suppose that $\left(X_{1}, \ldots, X_{n}\right)$ possesses a density $P_{n}\left(X_{1}, \ldots, X_{n} \mid \theta\right)$ with respect to a $\sigma$-finite measure $\mu_{n}$. Let $\hat{\theta}_{n}=\hat{\theta}_{n}\left(X_{1}, \ldots, X_{n}\right)$ be an MLE-a value of $\theta$ maximizing the likelihood function $P_{n}\left(X_{1}, \ldots, X_{n} \mid \theta\right)$ (uniqueness of $\hat{\theta}_{n}$ is not essential).

We put $L_{n}(\theta)=\log P_{n}\left(X_{1}, \ldots, X_{n} \mid \theta\right)$ and use $P_{\theta}$ and $E_{\theta}$ to denote probabilities and expectations computed under the assumption that $\theta$ is the true parameter value.

Let $\theta$ have an absolutely continuous prior distribution over $\Theta$, with density $\pi(\theta)$ with respect to Lebesgue measure. Then $\theta$ has an absolutely continuous posterior distribution with 
density

$$
\pi_{n}\left(\theta \mid X_{1}, \ldots, X_{n}\right)=\pi(\theta) P_{n}\left(X_{1}, \ldots, X_{n} \mid \theta\right) / P_{n}\left(X_{1}, \ldots, X_{n}\right),
$$

where $P_{n}\left(X_{1}, \ldots, X_{n}\right)=\int_{\Theta} \pi(\theta) P_{n}\left(X_{1}, \ldots, X_{n} \mid \theta\right) d \theta$.

Suppose that $\theta_{0}$ is an interior point of $\Theta$. We impose the following regularity conditions throughout:

(A1) The prior density $\pi(\theta)$ is continuous and positive at $\theta_{0}$.

(A2) $L_{n}(\theta)$ is a.s. twice continuously differentiable with respect to $\theta$ in some neighbourhood of $\theta_{0} \subset \Theta, N_{0}$ say.

(A3) $\left[-L_{n}^{\prime \prime}\left(\theta_{0}\right)\right] \rightarrow \infty$ a.s. $\left(P_{\theta_{0}}\right)$ as $n \rightarrow \infty$.

(A4) For any $\delta>0$ for which $N_{0}(\delta)=\left(\theta_{0}-\delta, \theta_{0}+\delta\right) \subset \Theta$ there exists $k(\delta)>0$ and $h(\delta) \downarrow 0$ as $\delta \downarrow 0$ satisfying

$$
\liminf _{n \rightarrow \infty} P_{\theta_{0}}\left\{\sup _{\theta \notin \Theta \backslash N_{0}(\delta)}\left[-L_{n}^{\prime \prime}\left(\theta_{0}\right)\right]^{-1}\left[L_{n}(\theta)-L_{n}\left(\theta_{0}\right)\right]<-k(\delta)\right\} \geqslant 1-h(\delta) .
$$

(A5) Given $\varepsilon>0$, there exists $\delta=\delta(\varepsilon)$ satisfying

$$
\liminf _{n \rightarrow \infty} P_{\theta_{0}}\left\{\sup _{\theta \in N_{0}(\delta)}\left|\left[L_{n}^{\prime \prime}\left(\hat{\theta}_{n}\right)\right]^{-1}\left(L_{n}^{\prime \prime}(\theta)-L_{n}^{\prime \prime}\left(\hat{\theta}_{n}\right)\right)\right|<\varepsilon\right\} \geqslant 1-\varepsilon .
$$

Conditions (A1) and (A2) require no comment while Condition (A3) essentially ensures that the process does not degenerate. Condition (A4) is little more than a simple sufficient condition for the consistency of $\hat{\theta}_{n}$ for $\theta_{0}$ while Condition (A5) is a continuity condition for $L_{n}^{\prime \prime}$. The Conditions A2-A5 are, in fact, implied by familiar classical conditions of Wald (1948) for consistency of the MLE, provided that it is unique. This can be straightforwardly checked via the conditions $A$ in Basawa et al. (1976).

We are now in a position to establish the following theorem, the proof of which is deferred until the following section.

Theorem. Suppose that conditions $A$ hold. Let $\sigma_{n}$ be the positive square root of $\left\{-L_{n}^{\prime \prime}\left(\hat{\theta}_{n}\right)\right\}^{-1}$ whenever this exists (which will be true with probability tending to unity as $n \rightarrow \infty$ by (A3) and (A5)). Then, if $-\infty \leqslant b<a \leqslant \infty$, the posterior probability that $\hat{\theta}_{n}+b \sigma_{n}<\theta<\hat{\theta}_{n}+a \sigma_{n}$, namely

$$
\int_{\hat{\theta}_{n}+b \sigma_{n}}^{\hat{\theta}_{n}+a \sigma_{n}} \pi_{n}\left(\theta \mid X_{1}, \ldots, X_{n}\right) d \theta
$$

tends in $P_{\theta_{0}}$ probability to

$$
(2 \pi)^{-\frac{1}{2}} \int_{a}^{b} e^{-\frac{1}{2} u^{2}} d u
$$

as $n \rightarrow \infty$.

This theorem provides the Bayesian analogue of confidence intervals. These are, of course, typically approached via asymptotic normality results for $\hat{\theta}_{n}$ in the Neyman-Pearson theory. Consequently, the assumptions underlying the result of the theorem should be contrasted with those necessary to ensure the asymptotic normality of $\hat{\theta}_{n}$, when appropriately normed, in a non-Bayesian situation.

The usual approach to asymptotic normality for $\hat{\theta}_{n}$ is via martingale theory and complicated regularity conditions are intrinsic (e.g. Heyde and Feigin (1975), Basawa et al. (1976), Heyde (1978). Indeed, under mild differentiability and integrability conditions,

$$
\left\{L_{n}^{\prime}(\theta)=\sum_{i=1}^{n} u_{i}(\theta), F_{n}, n \geqslant 1\right\},
$$

the $u$ 's being defined by differencing, is a martingale, $F_{k}$ denoting the $\sigma$-field generated by $X_{1}, \ldots, X_{k}$. Asymptotic normality of $\hat{\theta}_{n}-\theta$, when suitably normalized, holds under minor 
additional conditions if and only if a corresponding asymptotic result holds for $L_{n}^{\prime}(\theta)$. This, however, involves additional stringent assumptions on the increments $u_{i}(\theta)$ of the $L_{n}^{\prime}(\theta)$ process. Typical are conditions such as

$$
s_{n}^{-2} \sum_{1}^{n} E_{\theta}\left(u_{i}^{2}(\theta) \mid F_{i-1}\right) \rightarrow \eta^{2}(\theta) \text { in probability, }
$$

as $n \rightarrow \infty$ for some r.v. $\eta^{2}>0$ a.s. and

$$
s_{n}^{-2} \sum_{1}^{n} E_{\theta}\left(u_{i}^{2}(\theta) I\left(\left|u_{i}(\theta)\right|>\varepsilon s_{n}\right)\right) \rightarrow 0
$$

as $n \rightarrow \infty$ for any $\varepsilon>0$. Here $s_{n}^{2}=\Sigma_{1}^{n} E_{\theta} u_{i}^{2}(\theta)$. Requirements of this kind are indeed stringent by comparison with their rather robust Bayesian counterparts. There are not even any formal requirements concerning existence of moments in the Bayesian formulation.

The theorem is given for the single parameter case to facilitate comparison with corresponding results based on the MLE. These are not yet available in such general form in the multiparameter case. There is, however, no special difficulty in extending the theorem to the multiparameter case and details are provided in Johnstone (1978).

The scope of the theorem may also be extended in another direction, namely to increase somewhat the family of distributions to which the results are applicable. Thus, using a formulation along the lines of Dawid (1970) it is possible to deal with some distributions whose range depends on the parameter. There is, however, a significant cost in terms of formal complications of the regularity conditions.

\section{Proof of the TheOrem}

Write

$$
P_{n}\left(X_{1}, \ldots, X_{n} \mid \theta\right)=P_{n}\left(X_{1}, \ldots, X_{n} \mid \hat{\theta}_{n}\right) \exp \left\{L_{n}(\theta)-L\left(\hat{\theta}_{n}\right)\right\} .
$$

Whenever $\theta, \hat{\theta}_{n} \in N_{0}$ we have by Taylor expansion that

$$
L_{n}(\theta)=L_{n}\left(\hat{\theta}_{n}\right)+\frac{1}{2}\left(\theta-\hat{\theta}_{n}\right)^{2} L_{n}^{\prime \prime}\left(\theta_{n}^{*}\right)
$$

for $\theta_{n}^{*}=\theta+\gamma\left(\hat{\theta}_{n}-\theta\right)$ with $0<\gamma<1$. Thus, letting

$$
R_{n}=R_{n}\left(\theta, X_{1}, \ldots, X_{n}\right)=\sigma_{n}^{2}\left\{L_{n}^{\prime \prime}\left(\theta_{n}^{*}\right)-L_{n}^{\prime \prime}\left(\hat{\theta}_{n}\right)\right\},
$$

we have

$$
-\left(1-R_{n}\right) / \sigma_{n}^{2}=L_{n}^{\prime \prime}\left(\theta_{n}^{*}\right)
$$

so that, for $\theta, \hat{\theta}_{n} \in N_{0}$,

$$
P_{n}\left(X_{1}, \ldots, X_{n} \mid \theta\right)=P_{n}\left(X_{1}, \ldots, X_{n} \mid \hat{\theta}_{n}\right) \exp \left[-\left\{\left(\theta-\hat{\theta}_{n}\right)^{2} / 2 \sigma_{n}^{2}\right\}\left(1-R_{n}\right)\right] .
$$

Given $\varepsilon>0$ we choose $\delta=\delta(\varepsilon)$ so small that $N_{0}(\delta) \subset N_{0}$ and

$$
(1-\varepsilon)<\inf _{\theta \in N_{0}(\delta)} \pi(\theta) / \pi\left(\theta_{0}\right) \leqslant \sup _{\theta \in N_{0}(\delta)} \pi(\theta) / \pi\left(\theta_{0}\right)<1+\varepsilon,
$$

this last condition being justified by (A1). Now we split the integral defining $P_{n}\left(X_{1}, \ldots, X_{n}\right)$ into $I_{1}^{(n)}(\delta)$ and $I_{2}^{(n)}(\delta)$, taken over $\Theta \backslash N_{0}(\delta)$ and $N_{0}(\delta)$ respectively. Then, using (1),

$$
I_{1}^{(n)}(\delta)=P_{n}\left(X_{1}, \ldots, X_{n} \mid \hat{\theta}_{n}\right) \int_{\Theta \backslash N_{0}(\delta)} \pi(\theta) \exp \left\{L_{n}(\theta)-L_{n}\left(\hat{\theta}_{n}\right)\right\} d \theta
$$


so that, conditional on $L_{n}^{\prime \prime}\left(\hat{\theta}_{n}\right)<0$ and

$$
\begin{gathered}
A_{n}=A_{n}(\varepsilon)=\left[\sup _{\Theta \mid N_{0}(\delta)}\left\{-L_{n}^{\prime \prime}\left(\theta_{0}\right)\right\}^{-1}\left\{L_{n}(\theta)-L_{n}\left(\theta_{0}\right)\right\}<-k(\delta)\right], \\
{\left[P_{n}\left(X_{1}, \ldots, X_{n} \mid \hat{\theta}_{n}\right) \sigma_{n}\right]^{-1} I_{1}^{(n)}(\delta)} \\
=\exp \left\{L_{n}\left(\theta_{0}\right)-L_{n}\left(\hat{\theta}_{n}\right)\right\}\left\{-L_{n}^{\prime \prime}\left(\hat{\theta}_{n}\right)\right\}^{\frac{1}{2}} \times \int_{\Theta \backslash N_{0}(\delta)} \pi(\theta) \exp \left\{L_{n}(\theta)-L_{n}\left(\theta_{0}\right)\right\} d \theta \\
\leqslant
\end{gathered}
$$

by the definition of $\hat{\theta}_{n}$. Now

$$
\left\{-L_{n}^{\prime \prime}\left(\theta_{0}\right)\right\}^{\frac{1}{3}} \exp \left[-k(\delta)\left\{-L\left({ }_{n}^{\prime \prime} \theta_{0}\right)\right\}\right] \rightarrow 0 \text {, a.s. }
$$

as $n \rightarrow \infty$ in view of (A3) and hence, by virtue of (A3), (A5) and $P_{\theta_{0}}\left(L_{n}^{\prime \prime}\left(\hat{\theta}_{n}\right)<0\right) \rightarrow 1$ as $n \rightarrow \infty$, it follows that conditional on $A_{n}$ and

$$
B_{n}=B_{n}(\varepsilon)=\left\{\sup _{\theta \in N_{0}(\delta)}\left|\left[L_{n}^{\prime \prime}\left(\hat{\theta}_{n}\right)\right]^{-1}\left(L_{n}^{\prime \prime}(\theta)-L_{n}^{\prime \prime}\left(\hat{\theta}_{n}\right)\right)\right|<\varepsilon\right\},
$$

we have

$$
\left\{P_{n}\left(X_{1}, \ldots, X_{n} \mid \hat{\theta}_{n}\right) \sigma_{n}\right\}^{-1} I_{1}^{(n)}(\delta) \rightarrow 0,
$$

in probability.

To deal with $I_{2}^{(n)}(\delta)$ we first suppose that $\hat{\theta}_{n} \in N_{0}(\delta) \subset N_{0}$ and use (3) to write

$$
\left\{P_{n}\left(X_{1}, \ldots, X_{n} \mid \hat{\theta}_{n}\right) \pi\left(\theta_{0}\right)\right\}^{-1} I_{1}^{(n)}(\delta)=\int_{N_{0}(\delta)}\left\{\pi(\theta) / \pi\left(\theta_{0}\right)\right\} \exp \left[-\left\{\left(\theta-\hat{\theta}_{n}\right)^{2} / 2 \sigma_{n}^{2}\right\}\left(1-R_{n}\right)\right] d \theta .
$$

Then, putting

$$
I_{3}^{(n)}(\delta)=\int_{N_{0}(\delta)} \exp \left[-\left\{\left(\theta-\hat{\theta}_{n}\right)^{2} / 2 \sigma_{n}^{2}\right\}\left(1-R_{n}\right)\right] d \theta,
$$

we have from (4) that

$$
(1-\varepsilon) I_{3}^{(n)}(\delta)<\left\{P_{n}\left(X_{1}, \ldots, X_{n} \mid \hat{\theta}_{n}\right) \pi\left(\theta_{0}\right)\right\}^{-1} I_{2}^{(n)}(\delta)<(1+\varepsilon) I_{3}^{(n)}(\delta) .
$$

If $\sup _{N_{0}(\delta)}\left|R_{n}\right|<\varepsilon<1$,

$$
\int_{N_{0}(\delta)} \exp \left\{-\left(\theta-\hat{\theta}_{n}\right)^{2}(1+\varepsilon) / 2 \sigma_{n}^{2}\right\} d \theta<I_{3}^{(n)}(\delta)<\int_{N_{0}(\delta)} \exp \left\{-\left(\theta-\hat{\theta}_{n}\right)^{2}(1-\varepsilon) / 2 \sigma_{n}^{2}\right\} d \theta
$$

whilst for $\eta=+\varepsilon$ or $-\varepsilon$,

$$
\begin{aligned}
\int_{N_{0}(\delta)} \exp \left\{-\left(\theta-\hat{\theta}_{n}\right)^{2}(1+\eta) / 2 \sigma_{n}^{2}\right\} d \theta \\
=(2 \pi)^{\frac{1}{2}} \sigma_{n}(1+\eta)^{-\frac{1}{2}}\left[\Phi\left\{\sigma_{n}^{-1}\left(\theta_{0}+\delta-\hat{\theta}_{n}\right)(1+\eta)^{\frac{1}{k}}\right\}-\Phi\left\{\sigma_{n}^{-1}\left(\theta_{0}-\delta-\hat{\theta}_{n}\right)(1+\eta)^{\frac{1}{k}}\right\}\right],
\end{aligned}
$$

where

$$
\Phi(y)=(2 \pi)^{-\frac{1}{2}} \int_{-\infty}^{y} e^{-\frac{1}{2} u^{2}} d u .
$$

Conditions (A3) and (A5) imply that $\sigma_{n}^{-1} \rightarrow \infty$, in probability, and, since $\hat{\theta}_{n}$ is consistent, the factor in curly brackets in (6) converges to unity in probability. Thus, using (A5) to secure the appropriate bound on $R_{n}$, it follows that

$$
P_{\theta_{0}}\left((2 \pi)^{\frac{1}{2}}(1+\varepsilon)^{-\frac{1}{1}}<\sigma_{n}^{-1} I_{3}^{(n)}(\delta)<(2 \pi)^{\frac{1}{t}}(1-\varepsilon)^{-\frac{1}{2}} \mid A_{n} \cap B_{n}\right) \rightarrow 1
$$


as $n \rightarrow \infty$. The conditions (5), (6) and (8) then give

$$
P_{\theta_{0}}\left(C_{n} \mid A_{n} \cap B_{n}\right) \rightarrow 1
$$

as $n \rightarrow \infty$, where

$$
C_{n}=\left[(1+\varepsilon)^{\frac{1}{2}}(1-\varepsilon)<\left\{P_{n}\left(X_{1}, \ldots, X_{n} \mid \hat{\theta}_{n}\right) \pi\left(\theta_{0}\right)(2 \pi)^{\frac{1}{2}} \sigma_{n}\right\}^{-1} P_{n}\left(X_{1}, \ldots, X_{n}\right)<(1+\varepsilon)(1-\varepsilon)^{-\frac{1}{2}}\right] .
$$

But, by virtue of (A4) and (A5), and using a bar to denote complementation,

$$
\begin{aligned}
\underset{n \rightarrow \infty}{\liminf } P_{\theta_{0}}\left(A_{n} \cap B_{n}\right) & =1-\underset{n \rightarrow \infty}{\limsup } P_{\theta_{0}}\left(\bar{A}_{n} \cup \bar{B}_{n}\right) \\
& \geqslant 1-\limsup _{n \rightarrow \infty} P_{\theta_{0}}\left(\bar{A}_{n}\right)-\limsup _{n \rightarrow \infty} P_{\theta_{0}}\left(\bar{B}_{n}\right) \geqslant 1-h(\delta(\varepsilon))-\varepsilon,
\end{aligned}
$$

and hence,

$$
\begin{aligned}
\liminf _{n \rightarrow \infty} P_{\theta_{0}}\left(C_{n}\right) & \geqslant \liminf _{n \rightarrow \infty} P_{\theta_{0}}\left(C_{n} \cap A_{n} \cap B_{n}\right) \\
& =\liminf _{n \rightarrow \infty} P_{\theta_{0}}\left(C_{n} \mid A_{n} \cap B_{n}\right) P_{\theta_{0}}\left(A_{n} \cap B_{n}\right) \\
& \geqslant 1-h(\delta(\varepsilon))-\varepsilon .
\end{aligned}
$$

The results (9) holds for all $\varepsilon$ (and hence $h(\delta)$ ) small enough and thus,

$$
\left\{P_{n}\left(X_{1}, \ldots, X_{n} \mid \hat{\theta}_{n}\right) \sigma_{n}\right\}^{-1} P_{n}\left(X_{1}, \ldots, X_{n}\right) \rightarrow(2 \pi)^{\frac{1}{2}} \pi\left(\theta_{0}\right),
$$

in probability.

To complete the proof, we consider

$$
I_{2}^{\prime}=\int_{\hat{\theta}_{n}+b \sigma_{n}}^{\hat{\theta}_{n}+a \sigma_{n}} \pi(\theta) P_{n}\left(X_{1}, \ldots, X_{n} \mid \theta\right) d \theta,
$$

which is $I_{2}^{(n)}(\delta)$ with $N_{0}(\delta)$ replaced by $\left(\hat{\theta}_{n}+b \sigma_{n}, \hat{\theta}_{n}+a \sigma_{n}\right)$. Similarly, let $I_{3}^{\prime}$ be $I_{3}^{(n)}(\delta)$ with the limits of integration changed to $\left(\hat{\theta}_{n}+b \sigma_{n}, \hat{\theta}_{n}+a \sigma_{n}\right)$.

We use (Al) and the argument up to (6) so that if $\varepsilon>0$ is given, there exists $\delta$ such that if $\left(\hat{\theta}_{n}+b \sigma_{n}, \hat{\theta}_{n}+a \sigma_{n}\right) \subset N_{0}(\delta)$,

$$
(1-\varepsilon) I_{3}^{\prime}<\left\{P_{n}\left(X_{1}, \ldots, X_{n} \mid \hat{\theta}_{n}\right) \pi\left(\theta_{0}\right)\right\}^{-1} I_{2}^{\prime}<(1+\varepsilon) I_{3}^{\prime},
$$

while the right-hand side corresponding to (7) becomes

$$
(2 \pi)^{\frac{1}{2}} \sigma_{n}(1+\eta)^{-\frac{1}{2}}\left[\Phi\left\{a(1+\eta)^{\frac{1}{2}}\right\}-\Phi\left\{b(1+\eta)^{\frac{1}{2}}\right\}\right] .
$$

Clearly

$$
\lim _{n \rightarrow \infty} P_{\theta_{0}}\left\{\left(\hat{\theta}_{n}+b \sigma_{n}, \hat{\theta}_{n}+a \sigma_{n}\right) \subset N_{0}(\delta)\right\}=1
$$

and our previous argument can be paralleled to obtain

$$
\left\{P_{n}\left(X_{1}, \ldots, X_{n} \mid \hat{\theta}_{n}\right) \sigma_{n}\right\}^{-1} I_{2}^{\prime} \rightarrow(2 \pi)^{\frac{1}{2}} \pi\left(\theta_{0}\right)\{\Phi(a)-\Phi(b)\},
$$

in probability. Since

$$
\int_{\hat{\theta}_{n}+b \sigma_{n}}^{\hat{\theta}_{n}+a \sigma_{n}} \pi_{n}\left(\theta \mid X_{1}, \ldots, X_{n}\right) d \theta=I_{2}^{\prime} / P_{n}\left(X_{1}, \ldots, X_{n}\right),
$$

the result of the theorem follows from (10) and (11). 


\section{REFERENCES}

Basawa, I. V., Feigin, P. D. and Heyde, C. C. (1976). Asymptotic properties of maximum likelihood estimators for stochastic processes. Sankhyä A, 38, 259-270.

BASAWA, I. V. and SCOTT, D. J. (1976). Efficient tests for branching processes. Biometrika, 63, 531-536.

(1977). Efficient tests for stochastic processes. Sankhyä A, 39, 21-31.

Dawid, A. P. (1970). On the limiting normality of posterior distributions. Proc. Camb. Phil. Soc., 67, 625-633.

FeIGIN, P. D. (1978). The efficiency criteria problem for stochastic processes. Stoch. Proc. Appl., 6, 115-127

HEYDE, C. C. (1978). On an optimal property of the maximum likelihood estimator of a parameter from a stochastic process. Stoch. Proc. Appl. 8, 1-9.

Heyde, C. C. and Feigin, P. D. (1975). On efficiency and exponential families in stochastic process estimation. In Statistical Distributions in Scientific Work (G. P. Patil, S. Kotz and J. K. Ord, eds), Vol. 1, pp. 227-240. Dordrecht: Reidel.

JoHNSTONE, I. M. (1978). M.Sc. Thesis, Australian National University.

SweEting, T. J. (1978). On efficient tests for branching processes. Biometrika, 65, 123-127.

WALD, A. (1948). Asymptotic properties of the maximum likelihood estimate of an unknown parameter of a discrete stochastic process. Ann. Math. Statist., 19, 40-46.

WALKER, A. M. (1969). On the asymptotic behaviour of posterior distributions. J. R. Statist. Soc. B, 31, $80-88$. 